\title{
Distance Learning: Theory and Practice
}

\author{
L.A. Muraveva ${ }^{1 *}$ \\ ${ }^{1}$ Financial University under the Government of The Russian Federation, Department of Sociology, \\ History and Philosophy, Moscow, Russia
}

\begin{abstract}
This article discusses the process of active implementation of online learning modes based on digital technologies. Currently available theoretical postulates and recommended procedures of learning activities have been verified in practice during the course in History in Financial University under the Government of the Russian Federation. On this basis, certain experience of seminar classes has been accumulated (online conversations, discussions, presentations, webinars), this article is devoted to its analysis and generalization. The author has concluded that digital technologies diversify the classes and possess numerous tools to enhance motivation of students. The obtained results make it possible to state that application of several methods has been activated, such as brainstorming, mind games, metal mapping, interactive enquiries, testing and guessing games using learning platforms, for instance: Kahoot. Meanwhile, well balanced combination of the existing conventional learning modes and modern digital technologies makes it possible to provide even greater effect and result.
\end{abstract}

\section{Introduction}

The post-industrial society is based on information technologies, communications, and globalization. This article considers general theoretical provisions of distance learning, attempts to analyze and to share certain concepts in practice on the basis of the acquired, though not extensive, experience of presenting the course in History in Financial University under the Government of the Russian Federation, which, nevertheless, allows to highlight positive and negative aspects of wide-scale application of online technologies in learning activities of higher school regarding the humanities.

A modern teacher should have skills of e-learning. This was mentioned several times by Vladimir Putin, the President of the Russian Federation [1], in addition, this concept was also presented during conferencing with participation of members of Financial University [2, P. 39]. Ability of teachers to apply flexibly their personal qualities, as mentioned by Männikkö and Husu [3], is highly important for deep comprehension and development of learning activities.

As for now, there are numerous types and subtypes of information technologies: distance, electronic, virtual, network learning. While they are formally similar, each type is characterized by its peculiar features. Foreign researchers developed the system of e-learning

*Corresponding author: lam1812@mail.ru 
models [4-14]. The Russian scientists made their contribution to the development of this field, for instance, professors Andreev and Polat [15].

\section{Methods}

Personal experience of the author of this article regarding arrangement of distance learning is restricted by seminar classes. For convenient practical classes it is possible to apply various digital tools and interactive procedures: presentation, e-books, tests. Since seminars are aimed at consolidation of theoretical knowledge obtained during lectures and gaining skills of their practical application, then the didactic aims of such studies are as follows: to develop practical skills and habits, to provide creative environment for activation of mental activities of students, to form interest to R\&D activities, to control knowledge in certain topics and sections of academic program. Emotional and cognitive advantages of creativity and attentiveness in learning process were well analyzed, as Hensley wrote [16]. From this the main forms of studies are defined: frontal (one assignment for all students), group (the assignment is intended for a group of 5-6 students), and individual (individual assignment for each student). The author of this article uses all forms of learning during practical activities; however, the priority is given to frontal and individual forms. In the future the priorities should be changed, since the modern Generation $\mathrm{Z}$ is inclined to group activities.

Contrary to the conventional forms, the new learning technologies assume domination of game forms, simulation of situations, active interpersonal communication, new roles of a teacher, who acts as a tutor or a guide. The author of this article applies Scrabble game with students to reveal deeper insights into Russian history.

The range of the methods of mastering and consolidation of educational content is sufficiently versatile: conversations, recitations, discussions, brainstorming, or express test. Listening to reports and demonstration of presentations are widely applied with subsequent discussion in group. During one class it is allowed to apply limited number of methods. It should be mentioned that it is not recommended to apply all available methods at once. Firstly, quality deteriorates, secondly, acuteness and novelty of perception are mitigated upon further use, and finally, there is no sufficient time for application of all methods at once. Nowadays mental mapping is widely applied. This learning procedure can be used both synchronously (in classroom) or asynchronously. For instance, the author of this article proposed to the students to arrange mental map of periodization of Russian history as homework test.

Format of distance learning depends on respective online-platform selected by a higher school. All classes, tests, credits and attestations in Financial University are carried out using Microsoft Teams platform.

At present, various tools and services are available for online enquiries and online tests. They are comprised of MS Forms, Google Forms, Mentimeter, and others. The author of this article prefers to work with Kahoot platform. It is possible to carry enquiry on the basis of tests and quizzes. Though, free version provides two variants: enquiry with four answers, and True or False. The advantage of this service is that it is possible to set time for answers, and to obtain results immediately, there is always a winner and a certain rank of participants, which is practical in the case of rating system used in Financial University, and unlimited number of participants.

\section{Results}

As in conventional technologies, the aim and content of learning remain the same: results and quality. Learning principles gained certain supplements, tools and forms varied 
significantly. Under such conditions, a teacher should be able to create new educational space, which is a virtual medium. This is attributed to the necessity of interaction with students, which are not in the classroom but across the screen. In order to attract the interest of a student to study under such conditions, it is required to search for new ways of motivation, to make learning activities especially attractive. This important feature of learning was mentioned by prominent pedagogues Pestalozzi and Ushinsky [17]. This requires a lot of work from a teacher to develop nonstandard assignments aimed at brainwork and in-depth study of historical documents and scientific publications. Online learning is an open process, where conventional assignments cannot be applied. In the new environment, the main types of interaction and communications with teachers are consolations and analysis of work performed by a student. In such case grading marks also serve as a type of response aimed at development of students. Such approach increases specific weight of written communications with students, which increases time consumption and mental workload of a teacher. When no comments are made to the performed work, the students loss stimulus to perform and to present works in due time. This learning form increases the element of individualization in work of teacher with a student, as mentioned by Vaindorf-Sysoeva [18].

Krol, an expert in the field of modern learning activities, believes that "The focus in modern education is shifted to personal communications concerning significant issues, that is, the role of teacher is reduced to monitoring, tutoring, and not to creation and transfer of content. All content, which a student can receive independently, should be received remotely by any means. Therefore, in addition to correct content structure, it is necessary to pack education content so that it can be distributed remotely, automatically, and in asynchronous mode using modern technologies" [19].

In order to make seminar classes attractive for students and to achieve the required result, a teacher should preliminarily think through its organization. The author of this article keeps to the following procedure: generation of plan of upcoming class, timely sending it to students; definition of requirements to types of work; providing possibility to students to participate as leaders during the class; invitation of colleagues to participate in the seminar for further discussion of results and obtaining of fruitful criticism, recommendations, and exchange of experience. Presentation of students' reports should also be prepared: to propose topics; to formulate requirements; to determine time schedule and sequence of students' speeches; the students should be proposed to ask questions to the reporter. In this case it would be suitable to use chat, where the questions could be presented during reporting without interrogation of the reporter, the questions could be answered by the reporter after the presentation. Chat is a very useful tool for a teacher. It can be used to ask questions, to write comments, to follow activities of all students in the group.

Usage of service platforms for interactive enquiries, for testing, etc. requires for even more thorough preparation. Many tools require for preliminary registration, thus, a teacher should not only prepare program and questions, but also be aware of students' e-mails to register them, so that not to consume valuable time of seminar class for this routine activity.

\section{Conclusion}

Therefore, in a modern educational entity, implementation of digitalization is a necessary process, which would allow to move to a new level of management of learning quality, to improve efficiency of overall activities and reach competitive advantages in all areas. Under conditions of modern market economy, the main success of a higher school is the rate of its development, which provides for its competitiveness and attractiveness. The fourth technological revolution requires for implementation of new technology achievements upon training of modern personnel for all spheres of economy and society. International successfulness and competitiveness of Russia depend on solution to this problem. 
At the same time the work demonstrated that it would be highly erroneous to hope for and to absolutize only distance learning. These forms should be a tool to solve certain problems and should be combined with already verified conventional forms and procedures. The distance learning could be applied as exclusive in particular cases and situations related with environmental disasters, epidemics and other force majeure circumstances, as well as personal difficulties of a student or a teacher. Learning efficiency depends not only on written or verbal communication but also on direct human contact between a teacher and a student. Learning is accompanied by another important element of communication: behavioral education. It is implemented not only by means of didactic speeches and remarks. Behavioral education is performed by teacher's appearance, gestures and emotions, reaction to behavior of student in classroom, etc. In this case students acquire essential life lessons and behavioral models for a lifetime.

The experience of distance learning has obviously demonstrated that it is characterized by certain positive features. The coronavirus pandemic forced inquisitive and mature minds to consider reformation of the system of secondary and higher education. The optimum approach is to retain organic combination of conventional and distance learning technologies. Conversion to exclusively distance learning should be temporal, caused by extraordinary circumstances, such as the spring of 2020. Therefore, at present it is necessary to initiate development of a new doctrine of educational reform, including not only technologies but also legislative aspects. The future education would be successful only on the basis of proficient combination of previous and new achievements.

It is undisputable that the digital technologies made it possible to continue education activities and to complete the school year, to master the presented discipline in the required scope, to perform the course program. The author of this article believes that some disadvantages were caused by network malfunctions. Unfortunately, due to high load on Internet upon wide-scale conversion to online activities, network failures occurred more frequently. In this regard, spare variants should be provided. Thus, the author of this article appointed a student in each group, who acted as leader in the case of such failures and supported learning process until recovery of communication between the teacher and the group. In the higher school all classes should be recorded, hence, a teacher can always replay omitted fragments, review chat, highlight participants in conversation, and in the case of errors, write a letter with remarks and recommendation to the group e-mail. This is a definite advantage of digital technologies. A lot of work should be done aimed at implementation of digital technologies, for instance, the author of this article should master online presentation of lectures.

For further modernization and improvement of learning activities, it would be desirable to develop strategy of respective digital technologies. The strategy of application of distance technologies in educational entities should be an important part of the fourth technological revolution, which progresses right now. Such integrated computer system as digital technology, including numerous constituents from simulation to visualization and engineering analysis, should be applied more and more widely not only in industry but also during preparation of personnel for such companies and enterprises.

\section{References}

1. Putin: The formation of the digital economy is a matter of national security of the Russian Federation, TASS, July 5 (2017) Available at: https://tass.ru/ekonomika/4389411

2. A.A. Romanov, A paradigm shift in innovative product development: from disparate $R \& D$ to digital full-life projects, in Proceedings of the third conference "The economic potential of industry in the service of the military-industrial complex", Moscow, Russia 
(2017)

3. I. Männikkö, J Husu, Teach. Teach. Educ. 77, 126-137 (2019)

4. T. Maddison, M. Kumaran. (eds.), Distributed learning: pedagogy and technology in online information literacy instruction (Chandos Publishing, Oxford, 2017)

5. M. Dezouani, M. Fot, H. Hughes. (eds.)., Digital participation through social living labs (Chandos Publishing, Oxford, 2018)

6. J. Qin, Design requirements based on environmental modeling for software-intelligent systems (Morgan Kaufmann, Burlington, 2018)

7. W. Lawless, R. Mitt, S. Russell. (eds.)., Artificial intelligence for the Internet and everything else (Academic Press, New York, 2019)

8. K. Krishnan, Creating applications for working with big data (Academic Press, New York, 2019).

9. P. Sheldon, Ph. Rauschnabel, J. M. Honeycutt, The dark side of social networks. Psychological, managerial and social perspectives (Academic Press, New York, 2019)

10. D. Ladley, Data Management. How to develop, deploy and maintain an effective data management program (Academic Press, New York, 2019)

11. S. Bhunia, M. Tegranipur, Hardware protection. A practical approach to learning (Morgan Kaufmann, 2019).

12. N. Dey, Intelligent processing of speech signals (Academic Press, New York, 2019)

13. R. Park, E.J. de Visser, E. Rovira. Life with robots, New questions regarding the psychological and social consequences of robotic. (Academic Press, New York, 2019)

14. D.L. Hansen, B. Schneiderman, Analysis of social networks using NodeXL (Morgan Kaufmann, Burlington, 2020)

15. M.E. Vaindorf-Sysoeva, T.S. Gryaznova, V.A. Shitova. Methods of distance learning: textbook for universities (Moscow, Urait, 2018).

16. N. Hensley, J. Clean. Prod. 243, (2020)

17. P. Novikov, K. Ushinsky, Corf. Biographic Storytelling (Ural, Chelyabinsk, 1997)

18. M.E. Vaindorf-Sysoeva, Distance Learning: Simply about complex things. Institute of Online Education of the Financial University under the Government of the Russian Federation, Webinar 23 April (2020) Available at: https://www.youtube.com/watch?v=bSF4Ny99Jq0

19. A. Krol. Project Training, The 10th round table on the fundamental foundations of pedagogy and education. Transcript of the webinar December 2 (2016) Available at: https://medium.com/krol-institute/проектное-обучение-1daab521b4ff 